\title{
Relevance Ranking Metrics for Learning Objects
}

\author{
Xavier Ochoa $^{1}$ and Erik Duval ${ }^{2}$ \\ 1 Information Technology Center, Escuela Superior Politcnica del Litoral, \\ Va Perimetral Km. 30.5, Guayaquil - Ecuador \\ xavier@cti.espol.edu.ec \\ 2 Dept. Computerwetenschappen, Katholieke Universiteit Leuven, \\ Celestijnenlaan 200A, B-3001, Heverlee, Belgium \\ Erik.Duval@cs.kuleuven.be
}

\begin{abstract}
Technologies that solve the scarce availability of learning objects have created the opposite problem: abundance of choice. The solution to that problem is relevance ranking. Unfortunately current techniques used to rank learning objects are not able to present the user with a meaningful ordering of the result list. This work interpret the Information Retrieval concept of Relevance in the context of learning object search and use that interpretation to propose a set of metrics to estimate the Topical, Personal and Situational relevance. These metrics are calculated mainly from usage and contextual information. An exploratory evaluation of the metrics shows that even the simplest ones provide statistically significant improvement in the ranking order over the most common algorithmic relevance metric.
\end{abstract}

Key words: Learning Objects, Relevance Ranking, Topical Relevance, Personal Relevance, Situational Relevance

\section{Introduction}

In a broad definition, learning objects are any digital document with an educational objective. Learning Object Repositories (LOR) exist to enable sharing of such resources. To be included in a repository, learning objects are described by a metadata record provided at publishing time. This metadata can be manually or automatically generated [1]. Most LORs use the metadata information to implement keyword or field search functionality to allow the user to query the collection and retrieve relevant objects. Being any digital document, learning objects could be in any medium (text, pictures, audio, video, etc) and could be stored in any format. The metadata record helps to uniformly identify and describe the object and it is used as an object subrogate. The majority of learning object retrieval implementations is based on queries against the metadata fields stored into some form of database. On the other hand, Information Retrieval techniques based on the content of the object are rarer because of the diversity of the objects' medium and format. That is the reason why all initial LORs presented searchers with some kind of electronic form in which they were expected to translate their information need into search criteria for different fields. The 
LOR compares those values against the stored metadata records and return all the objects that satisfy the query. There was no specific order in which the objects were returned as it was expected that each would fulfill the information need of the user.

The approach based on the original metadata has been moderately successful for isolated repositories where the number of objects is small (in the order of thousands). A detailed query produces few results that the user could easily browse. But working with small, isolated repositories also meant that an important percentage of users did not find what they were looking for [2]. Current research in the Learning Object community has produced technologies and tools that solve the scarcity problem. Technologies as SQI [3] and OAI-PMH [4], enables to search several repositories simultaneously. Another technology, ALOCOM [5], decomposes complex learning objects into smaller components that are easier to reuse. Finally, automatic generation of metadata based on contextual information [6] allows the conversion of the learning content of Learning Management Systems (LMS), into metadata-annotated Learning Objects ready to be stored into a LOR. Although these technologies are solving the scarcity problem, they are creating an inverse problem, namely, abundance of choice.

Traditional retrieval mechanisms of LORs are no longer viable due to the abundance of learning objects. Even very detailed queries based on metadata fields will produce more results than the user is willing or able to browse. A proven solution for this problem is ranking or ordering the result list based on its relevance. To help the user to find relevant learning objects, Duval in [7] proposed the creation of LearnRank, an imaginary ranking function to access the relevance of learning objects similarly to how PageRank[8] access the relevance of web pages. Also, in a previous paper [9] the authors explore how Contextualized Attention Metadata [10] could be mined to obtain meaningful information about the relevance of a specific learning object for a specific user and context. The present work goes a step forward, proposing and testing a set of multi-dimensional relevance ranking metrics. This metrics will use external sources of information in addition to what is explicitly stated in the user query to provide a more meaningful relevance ranking than current query-matching implementations.

The structure of this paper is the following: Section 2 analyzes the different dimensions of the Information Retrieval's Relevance concept and their implications for Learning Object relevance ranking. These relevance dimensions are used as guidelines in Section 3 to propose a set of metrics that can be implemented to rank a list of learning objects based on usage and contextual information. To obtain a rough estimate of the benefit that these metrics could have in a real implementation, an exploratory study, where the metrics are compared against human relevance rankings, is performed in Section 4. The paper concludes with additional details about related and further work. 


\section{Relevance Ranking of Learning Objects}

The first step to build metrics to rank learning objects by their relevance is to understand what "relevance" means in the context of a Learning Object search. Borlund [11], after an extensive review of previous research on the definition of relevance for Information Retrieval, concludes that relevance is a multidimensional concept with no single measurement mechanism. Borlund defines four independent types of relevance: 'System' or 'Algorithmic' relevance which represents how well the query and the object match. Topical relevance that represents the relation between an object and the real-world topic of which the query is just a representation. 'Pertinence', 'Cognitive' or 'Personal' relevance which represents the relation between the information object and the information need that generate the query as perceived by the user. And 'Situational' relevance that represents the relation between the object and the work task that generated the information need.

These abstract relevance types need to be interpreted for the process and context of the learning object search:

- Algorithmic Relevance. In learning object search, the most common way to establish the algorithmic (or systemic) relevance is to compare the query parameters with the learning object metadata record. For example if the user searches for "inheritance", the algorithmic relevance of an object in the result list can be calculated as the frequency of times that the word "inheritance" appears in the title and description fields of the metadata record.

- Topical Relevance. In the context of learning objects the topic is strongly related to the Course / Lesson / Activity for which the learning objects will be used. For example if the user is searching for learning objects inside a course about Object Oriented Programming, the topical relevance of a learning object can be calculated as the number of times that the object has been used in similar courses.

- Personal Relevance. The personal (or cognitive or pertinance) relevance is directly derived from the information need as perceived by the user. In the context of learning, the perception of the information need is based on the teaching or learning style of the user. For example, a personal relevance ranking for a user that prefers highly interactive material should rank higher simulations and animations over text documents and slide presentations, even if all the presented objects have been used to learn the subject at hand.

- Situational Relevance. The task (and the task's context) that generates the user's information need determines the situational relevance of a result. Any task is normally aimed at reaching a specific learning objective, that is, to acquire a given knowledge, ability or competence. For example, a learning object with a very detailed explanation of how compilers work, should have different situational relevance ranking scores, depending on the specific public it is aimed towards. This holds true even if courses on the same subject are taught by the same professor to differnt student focus gruops. 
The information to estimate these relevance rankings is not only contained in the query parameters and the learning object metadata, but also in records of historical usage and the context where the query takes place. It is assumed that this information is available to the relevance ranker. This could seem unrealistic for classical Learning Object search, where the users, usually anonymous, perform their queries directly to the LOR through a web interface and the only information available are the query parameters. On the other hand, new implementations of LMSs, or plugins for old implementations such as Moodle [12] and BlackBoard [13], enable the capture of information by providing logged-in users with learning objects search capabilities as part of teacher workflow during the creation of courses and lessons.

While this interpretation of the relevance concept is exemplified with traditional or academic learning environment, it is at least as valid in less structured or informal settings such as corporate training or in-situ learning given that the environments used to assist such learning also store information about the topic searched by the user. This information takes te form of personal profiles, preferences, problem descriptions, previous and required competence.

A Ranking mechanism that could apply some combination of the abovementioned four types of relevance should provide the user with meaningfully ordered learning objects in the result list. The next section will propose pragmatic metrics that adjust to these different types of relevance in order to create a set of multidimensional relevance ranking metrics for learning objects.

\section{Ranking Metrics for Learning Objects}

To enable learning object search tools to exploit the different relevant ranking strategies described above, those strategies should be operationalized as ranking metrics. These metrics can be calculated automatically from existing or easily acquirable information. The metrics proposed here are inspired on methods currently used to rank other types of objects, for example ranking web pages, scientific papers, songs, and so forth. Different metrics for the same type of relevance will account for different levels of computational complexity and availability of usage and contextual information. Each metric is described by the raw data it requires and the algorithm to convert that data into concrete ranking values. These metrics, while not proposed as a complete or optimal way to compute the real relevance of learning object for a given user and task, are a clear first step to set a strong base-line implementation against which the effectiveness of more learning-specific metrics can be compared.

\subsection{Topical Relevance Ranking Metrics.}

Basic Topical Relevance Metric (BT). This metric makes two naïve assumptions. The first assumption is that each object in the result list is on-topic. Consequently, there is no need to explicitly define the topic of the query. The second assumption is that each object is relevant to just one topic. Accordingly, 
the degree of relevance of the object to the topic can be easily calculated by counting the number of times the object has been reused. Defining NC as the total number of courses of which the system keeps record, BT relevance metric is the sum of the times that the object is present in any of those courses (Equation 2). This metric is an adaptation of the Impact Factor metric [14] in which the relevance of a journal in a field is calculated simply counting the number of reference to papers in that journal during a given period of time.

$$
\begin{gathered}
\text { present }(\text { object }, \text { course })=\left\{\begin{array}{l}
1 ; \text { if object is published in course } \\
0 ; \text { otherwise }
\end{array}\right. \\
B T(\text { object })=\sum_{i=1}^{N C} \operatorname{present}_{\left(\text {object }, \text { course }_{i}\right)}
\end{gathered}
$$

Course-Similarity Topical Relevance Ranking (CST). In the context of learning object technologies, the course in which the learning object will be reused can be directly used as the topic of the query. Objects that are used in similar courses should be ranked higher in the list. In this metric, two courses are considered similar if they have a predefined percentage of learning objects in common. This relationship can be calculated constructing a 2-partite graph where courses are linked to objects published in them. This graph is folded over the object partition leaving a graph representing the existing relationships and strenghts between courses. The number of objects shared between two courses, represented in this new graph as the number of links between two coruses, determines the strenght of the relationship. The edges in the graph are then pruned according to the minimal strenght of relationship desired. The resulting connected sub-graph containing the course where the object will be inserted is taken as the new universe of courses (Figure 1). The ranking metric is calculated counting the number of times that a learning object in the list has been used in this new universe of courses of size NC (Equation 3). This metric is similar to the calculation made by e-commerce sites such as Amazon [15] where additionally to the current item, other items are recommended based on their probability of being bought together.

$$
C S T(\text { object })=\sum_{i=1}^{N C} \text { present }(\text { object }, \text { SimilarCourse } i)
$$

Internal Topical Relevance Ranking (IT). This is a refinement of the Basic Topical Relevance Rank based on the HITS algorithm [16] proposed to rank web pages. This algorithm states the existence of hubs, pages that point to other useful pages, and authorities, pages with information about a subject. The algorithm presumes that a good hub is a document that points to many good authorities, and a good authority is a document that many good hubs point to. In the context of learning objects, courses can be considered as hubs and learning 
Relevance Ranking Metrics for Learning Objects

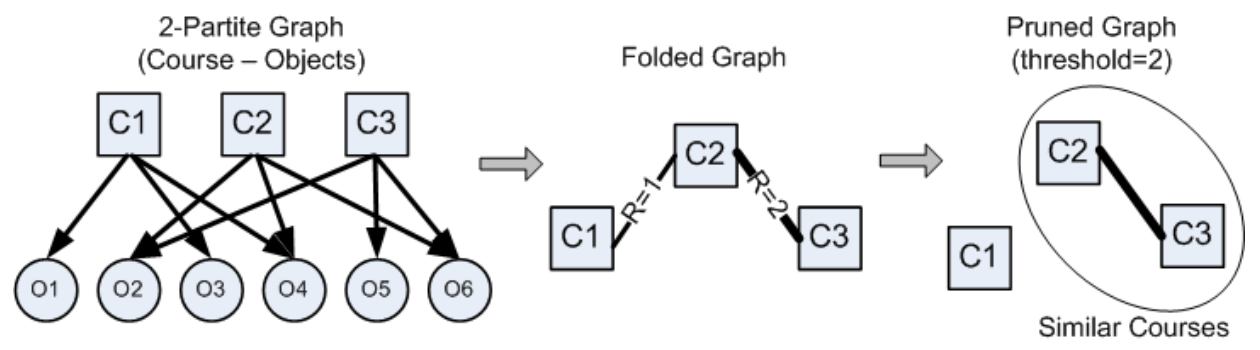

Fig. 1. Calculation of the Course-Similarity Topical Relevance Ranking (CST)

objects as authorities. To calculate the metric, a 2-partite graph is created with each object in the list linked to its containing courses. The hub value of each course is then calculated as the number of in-bound links that it has. Finally, the rank of each object is calculated as the sum of the hub value of the courses where it has been used (Equation 4).

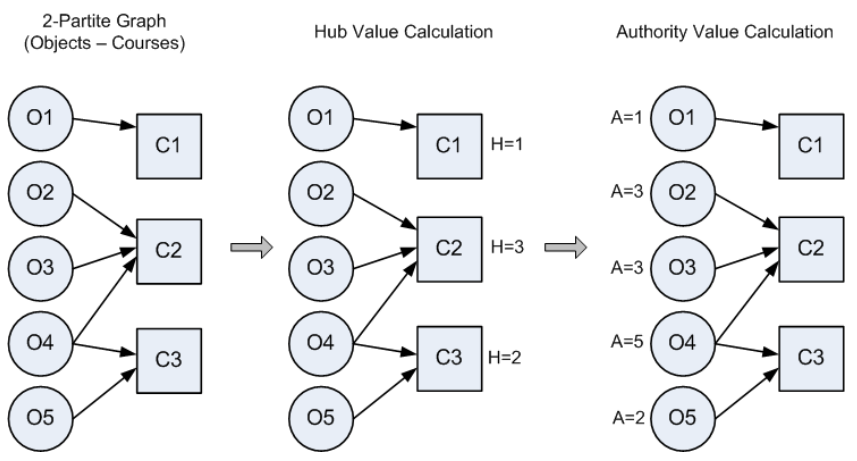

Fig. 2. Calculation of Internal Topical Relevance Ranking (IT)

$$
I T(\text { object })=\sum_{i=1}^{N} \operatorname{deg} \operatorname{ree}\left(\text { LinkedCourse }_{i}\right)=\text { authority }(\text { object })
$$

\subsection{Personal Relevance Ranking Metrics}

Basic Personal Relevance Ranking (BP). The easiest and least intrusive way to generate preference information is for users to analyze the objects they has used previously. First, for a given user, a set of the relative frequencies for the different metadata fields' values present in their objects is calculated (Equation 6 ). The number of these objects is defined by N. These frequencies are then 
compared with the metadata values of the objects in the result list. Finally, the relative frequency of the value present in the object is added to the personal relevance ranking value. This procedure is repeated for the NF selected fields the metadata standard (Equation 7). This methodology is especially useful for metadata fields' values based in a finite vocabulary. This metric is similar to that used for automatically recording TV programs in Personal Video Recorders [17]. The metadata of the programs watched by the user, such genre, actors, director and so forth, is averaged and compared against the metadata of new programs to select which ones will be recorded.

$$
\begin{aligned}
& \text { contains }(\text { object }, \text { field }, \text { value })=\left\{\begin{array}{l}
1 ; \text { if object'smetadata field }=\text { value } \\
0 ; \text { otherwise }
\end{array}\right. \\
& \text { freq }(\text { user }, \text { field }, \text { value })=\frac{1}{N} \sum_{i=1}^{N} \text { contains }(\text { object }(\text { user }, i), \text { field, value })
\end{aligned}
$$

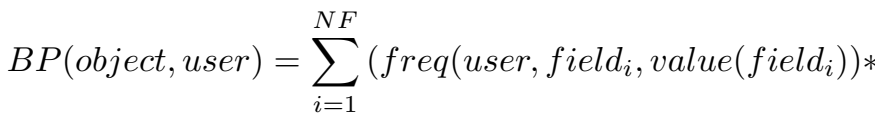

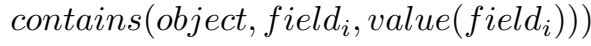

User-Similarity Personal Relevance Ranking (USP). Basic Personal Relevance Metric relies heavily on the metadata of the learning object in order to be effective. A more robust strategy to rank objects according to personal educational preferences is to find the number of times similar users have reused the objects in the result list. To find similar users, a process presented above to calculate the CST metric is followed. A 2-partite graph contains the objects linked to the users who have reused them. The graph is folded over the object partition and a relationship between the users is obtained. The graph can be pruned to obtain different levels of similarity. The remaining connected sub-graph, with NU nodes, which include the current user, is used to calculate the USP metric, as in Equation 9. The latter calculation is performed adding the number of times in which similar users have reused the object. This kind of metric is used, for example, by Last.fm and other music recommenders [18] who present new songs based on what similar users are listening to; similarity seen as the number of shared songs in their playlists.

$$
\begin{gathered}
\text { hasReused }(\text { object }, \text { user })=\left\{\begin{array}{l}
1 ; \text { ifobjecthasbeenreusedbyuser } \\
0 ; \text { otherwise }
\end{array}\right. \\
U S T(\text { object })=\sum_{i=1}^{N U} h a s U \text { sed }\left({\text { object } \left., \text { SimilarUser } s_{i}\right)}^{N}\right)
\end{gathered}
$$




\subsection{Situational Relevance Ranking Metrics}

Basic Situational Relevance Ranking (BS). Both the title and description of the course, lesson or activity in which the object will be inserted are sources of contextual information. Such information is usually written by the instructor to indicate to the students what the course, lesson or activity will be about. Keywords can be extracted from these texts and used to calculate a ranking metric based on the similarity between the keyword list and the content of the textual fields of the metadata record. To perform this calculation, a well known vector space retrieval algorithm [19] is used, which measures the distance between the M-dimensional vectors of the keyphrases extracted from the context and the terms present in the different text fields of the metadata record (Equation 10). One experimental version of this type of metric has been developed by Yahoo for the Y!Q service [20], that can perform contextualized searches based on the content of a web page in which the search box is located.

$$
B S(\text { object }, \text { context })=\frac{\sum_{i=1}^{M} \text { contextvector }_{i} * \text { objectvector }_{i}}{\sqrt{\sum_{i=1}^{M} \text { contextvector }_{i}^{2} \cdot \sum_{i=1}^{M} \text { objectvector }_{i}^{2}}}
$$

Course-Content Situational Relevance Ranking (CSS). To calculate this metric, other objects present in the course in which the object will be inserted are considered the contextual information. As with that proposed for the BP metric, the $\mathrm{N}$ objects contained in the course are "averaged" to create a set of relative frequencies for different fields of the learning object metadata record (Equation 11). This set of frequencies is then compared with the objects in the result list. The relative frequencies of the values present in the object's metadata are added to compute the final rank value (Equation 12).

$$
\begin{aligned}
& \text { freq }(\text { cour }, \text { field }, \text { value })=\frac{1}{N} \sum_{i=1}^{N} \operatorname{contains}(\text { object }(\text { cour }, i), \text { field }, \text { value })
\end{aligned}
$$

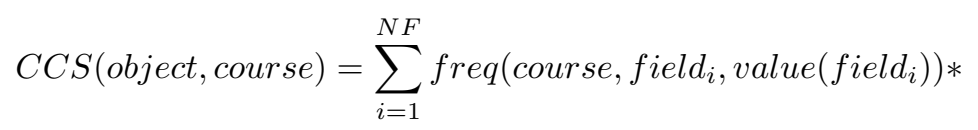

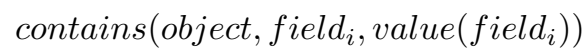

\section{Exploratory Experimentation}

In order to evaluate the potential impact the proposed metrics could have in the relevance ranking of learning object searches, an exploratory study has been performed, consisting of a small experiment in which subjects were asked to 
simulate the creation of a lesson inside a LMS. The subjects were required to rank the relevance of a list of learning objects, ranked using a algorithmic relevance metric, and to select from the list objects they consider appropriate for the lesson. The algorithmic relevance metric is compared with the subject's ranking to create a base line performance score. The proposed basic metrics for each one of the subjective relevance types are then used to reorder the list. Finally, the ranking scores are also compared against the subject's relevance score.

\subsection{Experimental Setup}

Ten users, eight professors and two research assistants from the Computer Science field, were required to create ten lessons related to different computer science concepts presented in Table 1 . In each lesson, the subjects were required to write a brief description of the lesson for hypothetical students. The subject was then presented with a list of ten objects. These objects were obtained from a LOR containing all PDF learning objects currently available in the MIT OCW website [21] (34,640 objects). This LOR was queried with a different query phrase for each lesson, as listed in Table 1. The title, description and keyword fields were text-matched with the query terms. The top-10 objects of each result list were used in the experiment. The subject then graded the relevance of each object to the lesson, to which end they used a 7 -value scale, from "Not relevant at all" to "Extremely Relevant". Moreover, subject's were required to select the objects they would include in the lesson. The data recollection was conducted using a Web application available at [22].

The initial rank of the objects was performed by the Lucene ranking algorithm, which is based on vector space retrieval [23]. This algorithm can be considered a good representation of current algorithmic relevance ranking. The basic topical relevance metric (BT) was calculated counting the number of times each object was selected to be included in the lesson. The selection of each subject was left out when compared against individual relevance evaluation. The basic personal relevance metric (BP) was calculated using historical information about the objects which subjects had published in their LMS courses. Three fields were captured: classification, document type and context level. These fields were selected on the basis of information available in the LOM record of the MIT OCW learning objects. The basic situational relevance ranking (BS) captured the text fed by the subjects into the description of the lesson. Any stopwords were eliminated and the resulting keywords were used to expand the query made to Lucene. The revised of the 10 objects was extracted then from the new result list.

Once all the metrics were calculated they were compared against the manual rank performed by the subjects. In order to measure the difference between the manual rank and each of the automated ranks, a variation of the Kendall tau metric [24] which deal with ties in the rank was used. This metric measure the distance between two permutations and is proportional to the number of swaps needed to convert one list into the other using bubble sort. If two ranks are 
Table 1. Task performed during the experiment and their correspondent query phrase

\begin{tabular}{|l|l|l|}
\hline$\#$ & Lesson to Create & Query Phrase \\
\hline 1 & Inheritance in object oriented languages & inheritance \\
\hline 2 & Algorithmic complexity & complexity \\
\hline 3 & Introduce the concept of computer networks & networks \\
\hline 4 & Introduce Human Computer Interaction concept & human computer interaction \\
\hline 5 & Explain tree structures & trees \\
\hline 6 & Xml markup & xml \\
\hline 7 & Introduce the concept of operating system & operating system \\
\hline 8 & Explain the artifical neural networks & neural networks \\
\hline 9 & How to normalize database tables & normalization \\
\hline 10 & Explain routing of packages in computer networks & routing \\
\hline
\end{tabular}

identical, the Kendall tau is equal to 0, if they are in inverse order, Kendall tau is equal to 1 .

\subsection{Results}

Only $12 \%$ of the objects presented to the users were manually ranked "Very Relevant"(5), "Highly Relevant"(6) or "Extremely Relevant"(7). This implies that pure algorithmic relevance ranking does a very bad job at providing relevant results to the user in the top-10 positions of the result list, especially if the repository contains a large amount of objects in different topics. Some searches, for example "human computer interaction" return almost "Not Relevant at All" results, even if in the repositories there were material for courses about Interface Design and Human Centered Computing.

The Kendall tau distance between the Base Rank (based on the Lucene algorithmic relevance metric) and the human has a mean value of 0.4 for all the searchers. For general query terms such as "trees"(5) and "human computer interaction" (6) it borders the 0.5 (meaning that there is no relation between both ranks). However, for very specific query terms such as "xml" (6) and "operating systems" (7) it provides a lower value, 0.3 implying a slight correlation between manual and automatic ranks. This is consistent with the low quality of the retrieval.

Table 2. Average distances between the manual ranking and the calculated metrics and the average improvement over the Base Rank

\begin{tabular}{|l|r|r|l|}
\hline Ranking Metric & Kendal tau & Improvement & Paired T-Test \\
\hline Lucene score & 0.4046 & - & \\
\hline Basic Topical & 0.2790 & $12.56 \%$ & $\mathrm{t}=9.50, \mathrm{df}=99, \mathrm{Sig}=0.000$ \\
\hline Basic Personal & 0.3392 & $6.53 \%$ & $\mathrm{t}=2.93, \mathrm{df}=99, \mathrm{Sig}=0.004$ \\
\hline Basic Situational & 0.3183 & $8.62 \%$ & $\mathrm{t}=6,424, \mathrm{df}=99, \mathrm{Sig}=0.000$ \\
\hline Linear Combination & 0.3139 & $9,07 \%$ & $\mathrm{t}=7.877, \mathrm{df}=99, \mathrm{Sig}=0.000$ \\
\hline
\end{tabular}


If the top-10 results provided by Lucene search engine are reordered using the basic topic relevance metric (BT), the Kendall tau distance is reduced by $12.6 \%$ on average compared with the Base Rank. When reordered with the basic personal relevance ranking (BP), the average Kendall tau distance reduction is $6.5 \%$. When the basic situational relevance ranking (BS) is uded, Kendall tau distance is reduced by $8.6 \%$. Finally, the simple linear combination of the metrics (topical, personal and situational) decrease the distance to $9 \%$ compared with the Base Rank. For more details on the results and their statistical significance, see Table 2 .

\subsection{Discussion of Results}

Basic topic relevance metric (BT) provides the best correlation with manual ranking. This result was expected because all the subjects participating in the experiment belong to the same field and were performing similar tasks. It fared better than the original ranking in all searches. It was the metric most directly related to human choice, as normally highly relevant items were selected for inclusion in the lessons.
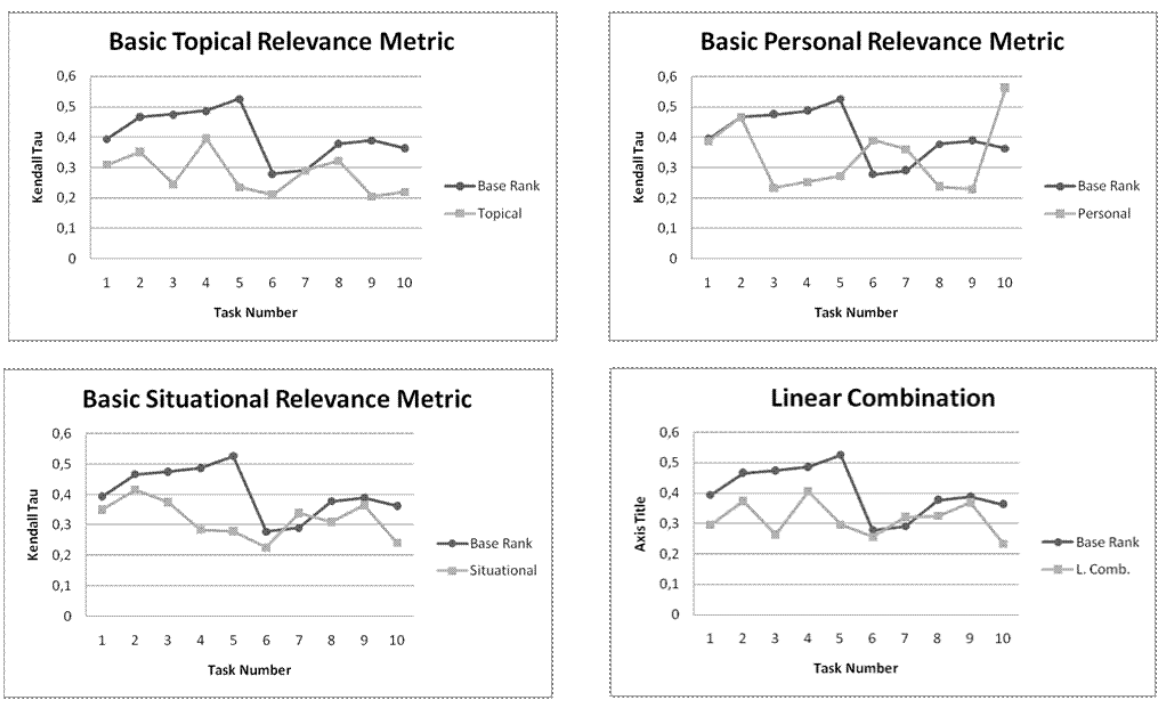

Fig. 3. Results of the Kendall tau distance from the manual ranking of the different metrics and their combination

Basic personal relevance metric (BP) presented some problems in certain queries. This can be explained as errors or unexpected values in the metadata 
records of the objects. While the object was relevant for a given lesson, metadata values do not always matched user preferences. For example, in search number 10 ("routing"), the topical classification of the objects were "Electrical Engineering", different from the "Computer Science" value that all the subjects had in their profile. Another case which exemplifies this problem was present in search number 1 ("inheritance"). The objects found more relevant came from a Programming course of the Civil Engineering department. This value was completely different from the value present in subject's profile.

Basic situational relevance metric (BS) provided an improvement in all but one search. It performed better for ambiguous query terms (note search number 4 and 5) while almost not affecting the performance of very specific query terms (searches 6 and 7 ). This result was expected given similar studies on query expansion using contextual descriptions.

To be useful in a comprehensive relevance ranking implementation, the ranks given by different metrics must be (linearly) combined and their estimating power should not be affected. The value obtained between the topical and contextual metrics results show that the combined rank behave well even if no weighting factors are included into the linear combination.

In conclusion, the basic ranking metrics always provide an average increase in the performance of the ranking compared with the Base Rank (Lucene textbased ranking). These results suggest that a full-fledge implementation of these metrics in a real environment will lead to a net benefit for final users searching for relevant learning objects.

\section{Related Work}

There are few published works on relevance ranking of learning objects, maybe because it was not seen as a critical problem in a world of small and isolated LORs. The work of Chellappa [25] represents the methodology followed by several repositories: adapting full-text search approaches to rank the learning objects based only on the similarity between the query terms and the text fields of the metadata record. This type of calculation only captures the Algorithmic relevance and does not provide the user with a real measurement of the object's Topical relevance, much less adapt the ranking for personal preferences or task. In contrast, the present work uses text similarity measurements only as a component of a more holistic approach to relevance estimation. Dolog et al. [26] propose a rule-based personalization based on the semantic description of both the user profile and the learning object. The main disadvantage of this approach is that it requires very rich metadata annotation of both the user and the object in order to work. Most of our metrics work with automatically created usage information and contextual information manually created as part of th original user workflow.

Taking another approach, Vargo et al propose [27] the use of data generated by user evaluation of the quality of the learning object to sort them into the result list. Users measure the LO quality using the Learning Object Review 
Instrument, a set of 9 quality parameters which the learning object should meet. The main drawback of this ranking approach is the well documented problem of the lack of scalability of user review. The authors of the present paper believe that this approach, while able to capture Topical relevance, could not be used to estimate Pertinence and Situational relevance. It is very difficult for reviewers to rate an object based in a different set of preferences or conditions than their own. While the Topical relevance estimation proposed in the present paper is not as reliable as a user review, nonetheless, it is scalable. Moreover, the use of personal and contextual information generates an adaptive ranking.

\section{Conclusion}

The main contribution of this work is the creation of a set of implementable ranking metrics which provide a comprehensive estimation of the multidimensional relevance of a learning object in realation to the user's information need. Usage and contextual information complement the information explicitly stated in both the query and the learning object metadata, in order to provide a more meaningful and robust ordering of the result list. This exploratory study confirmed that the application of even the simplest of these metrics leads to a statistically significant increase in performance compared to the currently most popular ranking method. While these metrics are not proposed as an optimal approach to the relevance ranking of learning objects they can be used to improve current learning objects search engines and set a strong baseline against which future learning-specific metrics could be tested.

\section{Further Work}

One of the purposes of this work is furtheer the discussion of better ways to help the user find relevant learning objects. In this line, more questions are raised than answered. Valid research topics unaddressed in this work include:

- Large Scale Implementation Which architectural compromises should be made to provide an acceptable computation time versus interoperability with current LMSs and LORs?.

- Federated Search. How should the metric calculation be adapted to environments in which only the top-k objects of each repository are known? Moreover, how shold the ranking made by different LORs be aggregated?

- Underlying theory. Are there deeper pedagogical or cognitive reasons which explain the success or failure of different metrics?

- Metric combination and Tuning. Is there an optimal relevance ranking function and if so, how should existing metrics be combined? Do different users prefer different combination of metrics?

The main task left for further work is to execute an empirical study with both a full implementation of the metrics and real users performing within their normal workflow. Once there is enough data collected, the user interaction with 
the system and the progress of the different metrics could be analyzed to shed light on these questions. We also hope that other researchers start proposing improvements to this initial approach.

\section{References}

1. Cardinaels, K., Meire, M., Duval, E.: Automating metadata generation: the simple indexing interface. In: WWW '05: Proceedings of the 14th international conference on World Wide Web, New York, NY, USA, ACM Press (2005) 548-556

2. Najjar, J., Klerkx, J., Vuorikari, R., Duval, E.: Finding appropriate learning objects: An empirical evaluation. In Rauber, A., Christodoulakis, S., Tjoa, A.M., eds.: ECDL. Volume 3652 of Lecture Notes in Computer Science., Springer (2005) 323-335

3. Simon, B., Massart, D., van Assche, F., Ternier, S., Duval, E., Brantner, S., Olmedilla, D., Miklos, Z.: A simple query interface for interoperable learning repositories. In Simon, B., Olmedilla, D., Saito, N., eds.: Proceedings of the 1st Workshop on Interoperability of Web-based Educational Systems, Chiba, Japan, CEUR (2005) 11-18

4. Herbert, M.L.N.: Resource harvesting within the oai-pmh framework. D-Lib Magazine $(\mathbf{1 0}(12))$

5. Verbert, K., Jovanovic, J., Gasevic, D., Duval, E.: Repurposing learning object components. In: OTM Workshops. (2005) 1169-1178

6. Ochoa, X., Cardinaels, K., Meire, M., Duval, E.: Frameworks for the automatic indexation of learning management systems content into learning object repositories. In Kommers, P., Richards, G., eds.: Proceedings of the ED-MEDIA 2005 World Conference on Educational Multimedia, Hypermedia and Telecommunications. (2005)

7. Duval, E.: Learnrank: the real quality measure for learning materials (2005)

8. Page, L., Brin, S., Motwani, R., Winograd, T.: The pagerank citation ranking: Bringing order to the web. Technical report, Stanford Digital Library Technologies Project (1998)

9. Ochoa, X., Duval, E.: Use of contextualized attention metadata for ranking and recommending learning objects. In: CAMA '06: Proceedings of the 1st international workshop on Contextualized attention metadata, New York, NY, USA, ACM Press (2006) 9-16

10. Najjar, J., Duval, E.: Attention metadata: Collection and management. In IEEE, ed.: WWW 2006, Edinburgh, Scotland (2006)

11. Borlund, P.: The concept of relevance in ir. Journal of the American Society for Information Science and Technology 54(10) (2003) 913-925

12. Broisin, J., Vidal, P., Meire, M., Duval, E.: Bridging the gap between learning management systems and learning object repositories: Exploiting learning context information. In: AICT-SAPIR-ELETE '05 (AICT/SAPIR/ELETE'05), Washington, DC, USA, IEEE Computer Society (2005) 478-483

13. Vandepitte, P., Van Rentergem, L., Duval, E., Ternier, S., Neven, F.: Bridging an lcms and an lms: a blackboard building block for the ariadne knowledge pool system. In: Proceedings of ED-MEDIA 2003 World Conference on Educational Multimedia, Hypermedia, and Telecommunications, AACE, AACE (2003) 423424 URL: http://go.editlib.org/p/13794. 
14. Dong, P., Loh, M., Mondry, A.: The "impact factor" revisited. Biomedical Digital Libraries 2(1) (2005) 7+

15. Linden, G., Smith, B., York, J.: Amazon.com recommendations: Item-to-item collaborative filtering. IEEE Internet Computing 7(1) (2003) 76-80

16. Kleinberg, J.M.: Authoritative sources in a hyperlinked environment. Journal of the ACM 46(5) (1999) 604-632

17. Pigeau, A., Raschia, G., Gelgon, M., Mouaddib, N., Saint-Paul, R.: A fuzzy linguistic summarization technique for tv recommender systems. In: IEEE Int. Conf. of Fuzzy Systems (FUZZ-IEEE'2003), St-Louis, USA (2003) 743-748

18. Upendra, S.: Social information filtering for music recommendation (1994)

19. Aizawa, A.: An information-theoretic perspective of tf-idf measures. Information Processing \& Management 39(1) (2003) 45-65

20. Kraft, R., Maghoul, F., Chang, C.C.: Y!q: contextual search at the point of inspiration. In: CIKM '05: Proceedings of the 14th ACM international conference on Information and knowledge management, New York, NY, USA, ACM Press (2005) $816-823$

21. MIT: (Opencourseware - ocw home, http://ocw.mit.edu/index.html)

22. Ochoa, X.: (On-line experiment application. user:test, password:test. http://ariadne.cti.espol.edu.ec/ranking)

23. Hatcher, E., Gospodnetic, O.: Lucene in Action (In Action series). Manning Publications Co., Greenwich, CT, USA (2004)

24. Fagin, R., Kumar, R., Mahdian, M., Sivakumar, D., Vee, E.: Comparing and aggregating rankings with ties. In: PODS '04: Proceedings of the twenty-third ACM SIGMOD-SIGACT-SIGART symposium on Principles of database systems, New York, NY, USA, ACM Press (2004) 47-58

25. Chellappa, V.: Content-based searching with relevance ranking for learning objects (2004)

26. Dolog, P., Henze, N., Nejdl, W., Sintek, M.: Personalization in distributed elearning environments. In: WWW Alt. '04: Proceedings of the 13th international World Wide Web conference on Alternate track papers \& posters, New York, NY, USA, ACM Press (2004) 170-179

27. Vargo, J. Nesbit, J.C.B.K.A.A.: Learning object evaluation: Computer-mediated collaboration and inter-rater reliability. Iternational Journal of Computers and Applications 25 (2003) 198-205 